\title{
CLINICAL EVALUATION OF PLATELET RICH FIBRIN VERSUS SUBEPITHELIAL CONNECTIVE TISSUE GRAFT, FOR SOFT TISSUE AUGMENTATION AROUND IMPLANT IN THE AESTHETIC ZONE: A RANDOMIZED CONTROL CLINICAL TRIAL
}

\author{
May Mohamed Kamal*, Weam Elbattawy**, Salma Eid ${ }^{* * * *}$ and Mona Shoeib****
}

\begin{abstract}
Background: the current study investigated platelet rich fibrin and subepithelial connective tissue graft in preserving crestal bone and soft tissue around delayed implants.

Methods: Twelve participants with thin gingival biotype, were randomly assigned in two equal parallel groups. Both received delayed implant, augmented by subepithelial connective tissue graft versus platelet rich fibrin. Crestal bone level was measured at 3,6 and 9 months. Gingival thickness and keratinized tissue width were measured at baseline, 3 and 6 months, pink esthetic score at 6 months, pain, swelling and patient satisfaction were reported after 7 days and at the end of the follow up period respectively.
\end{abstract}

Results: Both groups showed statistically significant improvement in all clinical outcomes at 3 and 6 months with no statistically significant difference at different observational periods. Subepithelial connective tissue graft showed statistically significant increase in gingival thickness and pink esthetic score compared to platelet rich fibrin after 6 months with no statistically significant difference regarding the crestal bone loss, keratinized tissue width, postoperative pain, swelling and overall patient satisfaction between both groups.

Conclusion: Inspite that PRF showed less values of crestal bone loss yet both treatment modalities could not prevent the postsurgical crestal bone loss to occur, with no statistically significant difference between them. Subepithelial connective tissue graft is effective at increasing the gingival thickness around delayed implants.

Clinical relevance: Both treatment modalities could be used safely around dental implant. Subepithelial connective tissue graft is more effective in thin phenotype for augmenting gingival thickness.

\footnotetext{
* M.Sc. of Periodontology, Faculty of Dentistry, Cairo University

** M.Sc, Phd, Lecturer, Department of Oral Medicine and Periodontology, Faculty of Dentistry, Cairo University. *** M.Sc, Phd, Lecturer, Department of Oral and Maxillofacial Radiology, Faculty of Dentistry, Cairo University. **** M.Sc, Phd, Professor, Department of Oral and Maxillofacial Radiology, Faculty of Dentistry, Cairo University.
} 


\section{INTRODUCTION}

Dental implants have been considered a good option for partially edentulous patients in maintaining healthy mucosa with minimal crestal bone loss and no extensive harm to the adjacent natural teeth ${ }^{[1]}$. Delayed implants placed after complete healing of the ridge ensured a stable ridge dimension. However, the disadvantage of prolonged healing time and bone resorption needed additional augmentation procedure ${ }^{[2]}$.

The pink esthetic success was found to be dependent on different factors that involved the implant position, multiple implant placement and the soft tissue management manner. Two mm of facial bone thickness has been suggested as a minimum to prevent future recession, which would potentially expose implant collars and lead to loss of the harmonious gingival margin ${ }^{[3]}$.

Enough keratinized tissue was considered important for marinating healthy tissue around dental implant ${ }^{[4]}$. Also soft tissue biotype has been considered as a critical factor, where patients would show either thin scalloped gingiva or thick flat biotype ${ }^{[5]}$. Moreover, it has been proven that thin biotype had higher liability for gingival recession from any trauma during the surgical and prosthetic procedures in comparison to thick flat biotype and the underlying bone could suffer from rapid resorption in association with soft tissue recession ${ }^{[6]}$.

SCTG has been widely utilized aiming at increasing the width and thickness of keratinized tissue either around natural teeth or around implants for resisting and treating recession, masking the metallic implant color and also for papillary reconstruction ${ }^{[7]}$.

However, an additional harvesting site in autografts with its associated pain, morbidity, sometimes poor quality and limited amount of graft material limited their use. To overcome these problems, a large amount of alternative materials for soft tissue augmentation have been used and developed to date ${ }^{[8]}$.

PRF gained its popularity as a healing biomaterial for both soft and hard tissue because of the presence of various growth factors also it would facilitate tissue healing and regeneration by enhancing angiogenesis, chemotaxis, cell proliferation and differentiation and extracellular matrix synthesis ${ }^{[9,10]}$. For best outcomes in human tissue healing and regeneration, mutual interaction between a scaffold (fibrin matrix), platelets, growth factors, leukocytes, and stem cells are required [11, ${ }^{12]}$. Therefore, PRF could promote wound healing, bone growth and maturation, graft stabilization and hemostasis and even improve the handling properties of graft materials ${ }^{[13,14]}$.

\section{MATERIALS AND METHODS}

\section{Study design}

This study was designed as a parallel, randomized, controlled clinical trial to compare the clinical and radiographic outcomes of PRF to SCTG in maintaining crestal bone and enhancing soft tissue around delayed implants. The study protocol was registered in ClinicalTrials.gov (ID: NCT03219944.) and approved by The Research Ethics committee, Faculty of Dentistry, Cairo University (July 2017).

\section{Study population}

This investigation included 12 delayed implants placed in 12 patients ( 2 males and 10 females) with missing maxillary tooth in the esthetic zone and seeking its replacement. Subjects were selected from the outpatient clinic, Department of oral Medicine and Periodontology, Faculty of Dentistry, Cairo University between September 2017 and April 2018.

\section{Pretreatment}

Initial examination, including full mouth clinical and radiographic examination, was performed. 
A preoperative cone beam computed tomography (CBCT) was performed for each patient who met the inclusion criteria prior to the surgery to determine bone height and width and decide the implant length and diameter to be placed and to evaluate the underlying bone condition ${ }^{[15]}$.

\section{Randomization}

A computer-generated random allocation sequence was executed by external assistant who was not involved in the recruitment. Allocation concealment was achieved by sequentially numbered, opaque, sealed envelopes including the randomization code for each patient.

\section{Blinding}

The current trial was a single-blinded clinical trial. Blinding included the outcome assessor and the statistician. It was impossible for the operator and the participants to be blinded as the interventions were completely different.

\section{Radiographic parameters}

Standardized periapical digital radiographs were taken using PSP sensors (Digora ${ }^{\mathrm{TM}}$ PSP Imaging plate. SOREDEX Inc., Tuusula, Finland) size 1 or 2 with parallel technique using X-Ray Holder kit and custom-made bite block was fabricated for each case ${ }^{[16]}$.

Each implant had 8 readings for CBL: mesial and distal readings on the day of the intervention (baseline), 3, 6 and 9 months postoperatively. The crestal bone loss along the months was calculated as the difference between the initial measurement (baseline) and the measurement obtained at the three follow-up phases ${ }^{[17]}$ (Figure 1).

\section{Clinical Parameters}

Clinical parameters were measured at baseline, 3 and 6 months postoperatively. Clinical parameters included: gingival thickness (GT), keratinized tissue width (KTW) and pink esthetic score

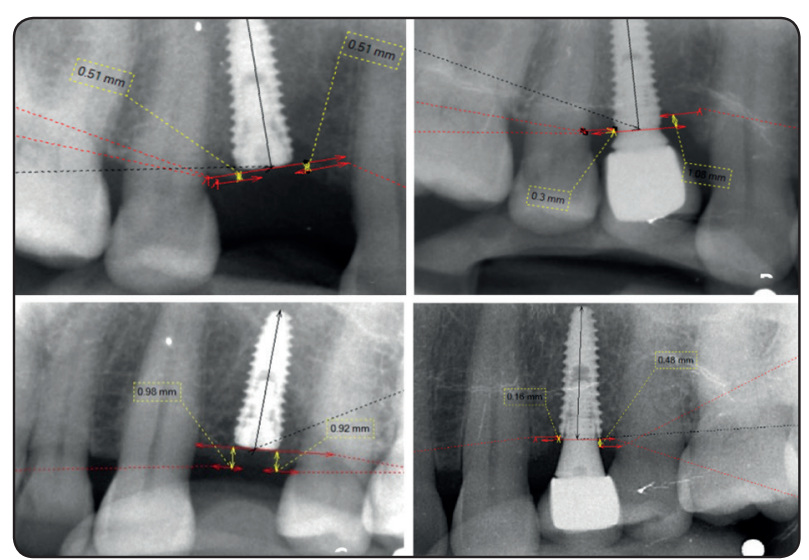

Fig. (1) (A) Digital periapical radiograph at the base line of control group (SCTG) showing CBL (B) Digital periapical radiograph at 9 months of control group (SCTG) showing CBL (C) Digital periapical radiograph at base line test group (PRF) showing CBL (D)Digital periapical radiograph at 9 months test group (PRF) showing CBL.

(PES), which were measured using William's graduated periodontal probe. GT was measured by transgingival-piercing of the tissues using an anesthetic needle with a rubber stopper $2 \mathrm{~mm}$ coronal to the MGJ and in the mid distance mesiodistally ${ }^{[18]}$. KTW was measured at the mid buccal area from the gingival crest to the MGJ. PES was measured at 6 months ${ }^{[19]}$.

\section{Patient reported outcomes}

Post-operative pain and swelling was assessed by the patient for the first week postoperatively ( 0 7 days) using Verbal Rating Scale (VRS) ${ }^{[20]}$.

At the end of the follow up period (9 months), assessment of the patient's satisfaction was done using Patient Satisfaction Questionnaire (PSPSQ) ${ }^{[21]}$.

\section{Treatment Protocols}

All procedures were done under completely aseptic conditions. Patients were anaesthetized by buccal and palatal infiltration. Crestal incision and full thickness mucoperiosteal flap was elevated buccally and lingually. Then the bone width was 
measured again using bone caliper to confirm the implant width as detected in CBCT. ${ }^{[22]}$. Sequential drilling started by the pilot drill till the last drill that suited the planned implant size. Before implant placement a parallel pin was used to check the implant parallelism. Implant insertion was done in the osteotomy site using torque wrench by selftapping fashion till the implant was placed $0.5-1 \mathrm{~mm}$ below the alveolar bone crest ${ }^{[23]}$ with adequate primary stability (torque about $35 \mathrm{~N}$ ) ${ }^{[24]}$. A split thickness pouch was created in the labial/buccal flap using blade \#15C where it was made parallel to the periosteum. The pouch was created to receive the graft and allow its fixation ${ }^{[25]}$.

Control group (SCTG): SCTG was harvested from the palate by single incision technique ${ }^{[26]}$. The SCTG was placed in the pouch over the recipient site below the labial/buccal flap and extending palatally. The graft was sutured in a horizontal mattress manner to the labial/buccal flap by 5-0 resorbable braided polyglycolic acid suture material. Flap approximation and primary wound closure was achieved with horizontal mattress and single interrupted manner ${ }^{[27]}$. The palatal wound, from which the SCTG was harvested, was sutured by sterilized, natural non absorbable silk 5-0 (Figure 2).

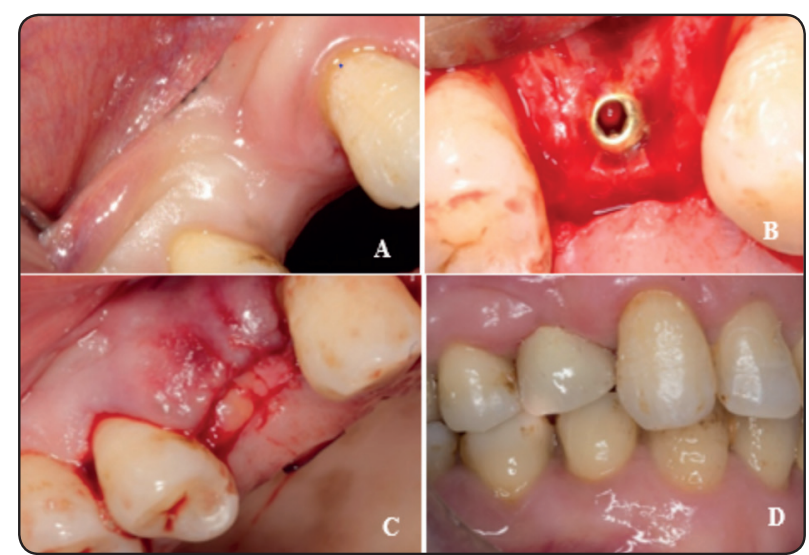

Fig. (2) (A) Preopearive ,(B) Implant placement,(C) placement of SCTG over implant, (D) 6 months postoperative.

\section{Test group (PRF):}

PRF was prepared by drawing $10 \mathrm{ml}$ of blood from the antecubital patient vein using butterfly/ needle winged infusion set and transferred to a glass test-tube without anticoagulant. The blood sample was immediately centrifuged at $3000 \mathrm{rpm}$ for $10-12$ min in an electric centrifuge[17]. Then PRF layer that needed was separated. Then the immediately prepared PRF would be squeezed between two sterile glass laps to flatten it into a membrane form. A double-layered membrane of PRF was placed in the pouch over the recipient site below the labial/buccal flap and extending palatally without suturing. Flap approximation was performed for wound closure and sutured in the same manner as control group (Figure 3).

\section{Post-surgical phase:}

Systemic antibiotics (Amoxicillin 1g) were prescribed b.i.d. for 5 days to prevent any chance of post-operative infection ${ }^{[28]}$. Anti-inflammatory drugs (Ketoprofen $100 \mathrm{mg}$ ) was administrated every 12 hours to avoid any chance of edema, pain or swelling ${ }^{[28]}$. Antiseptic mouth rinse $0.12 \%$ chlorhexidine HCL was prescribed for 60 seconds two times per day for 14 days ${ }^{[29]}$. Patient self-care instructions were emphasized on avoiding any

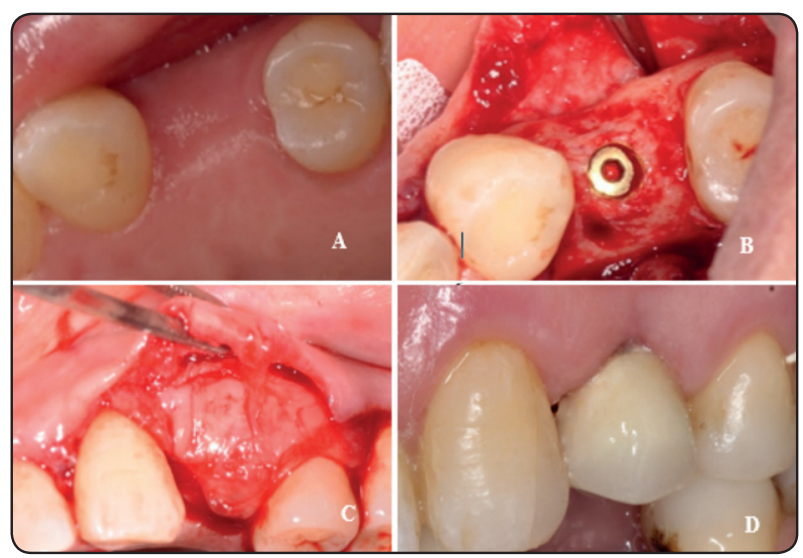

Fig. (3) (A) Clinical preopearive photo, (B) Implant placement, (C) Placement of double layer PRF over implant, (D) 6 months postoperative. 
vigorous brushing and trauma to the surgical site for one week ${ }^{[30]}$. Sutures were removed fourteen days postsurgically.

\section{Statistical \& Power analysis:}

Values were presented as mean and standard deviation (SD) values. Data were explored for normality using Kolmogorov-Smirnov test of normality. For parametric data (GT, KTW and PES), independent $t$ test was used to compare both groups, while ANOVA test (followed by Tukey's post hoc test) were used for comparison between different observations within the same group. For nonparametric data, (crestal bone loss) Mann Whiney $\mathrm{U}$ test was used to compare both groups. Categorial data (Pain and response to questionnaire) were compared using chi square test. The significance level was set at $p<0.05$. Statistical analysis was performed with SPSS 18.0 (Statistical Package for Scientific Studies, SPSS, Inc., Chicago, IL, USA) for Windows.

\section{RESULTS}

\section{Radiographic parameters}

The mean and standard deviation of CBL recorded for both groups throughout the study is represented in table (1), while figure (1) represents the preoperative and postoperative radiographs after 9 months for the two groups. Comparing the two groups, there was greater mean crestal bone loss values in the SCTG group, however, the results in the follow up 3, 6 and 9 months showed no statistically significant difference between both groups $(P=0.150,0.262$ and 0.261$)$ respectively.

\section{Clinical parameters}

Table (2) shows the clinical parameters recorded for both groups throughout the study. On comparing both groups, it was observed that greater mean values were recorded in the SCTG group at all observation. However, at 6 months the SCTG group showed a statistically significant increase $(P=0.016)$ in mean GT compared to the PRF group.

The difference in the mean values of KTW on comparing both studied groups at baseline, 3 and 6 months showed no statistically significant difference postoperatively $(P$-value $=0.421,0.401$ and 0.154 respectively).

The esthetic outcomes were measured by the PES at 6 months postoperatively and showed greater mean values for the SCTG group in comparison to the PRF group with statistically significant difference between the both groups $(P$-value $=$ $0.035)$.

TABLE (1): Results of crestal bone level (CBL) in both studied groups throughout the experimental period.

\begin{tabular}{ccccc}
\hline CBL mean $( \pm$ SD) & SCTG & PRF & Mean difference CI $(95 \%)$ & P-value \\
\hline Baseline & $0.58^{\mathrm{c}}( \pm 0.16)$ & $0.51^{\mathrm{d}}( \pm 0.07)$ & & \\
3 months & $1.78^{\mathrm{b}}( \pm 0.22)$ & $1.45^{\mathrm{c}}( \pm 0.40)$ & & \\
6 months & $1.95^{\mathrm{b}}( \pm 0.29)$ & $1.71^{\mathrm{b}}( \pm 0.36)$ & & \\
9 months & $2.28^{\mathrm{a}}( \pm 0.28)$ & $2.06^{\mathrm{a}}( \pm 0.14)$ & & \\
p-value & $<0.001^{*}$ & $<0.001^{*}$ & & \\
\hline Baseline-3 months & $1.20( \pm 0.22)$ & $0.95( \pm 0.39)$ & $0.25[-0.17,0.68]$ & $0.150 \mathrm{~ns}$ \\
Baseline-6 months & $1.37( \pm 0.28)$ & $1.20( \pm 0.36)$ & $0.17[-0.25,0.59]$ & $0.262 \mathrm{~ns}$ \\
Baseline-9 months & $1.70( \pm 0.20)$ & $1.55( \pm 0.15)$ & $0.15[-0.08,0.38]$ & $0.261 \mathrm{~ns}$ \\
\hline
\end{tabular}

Significance level $\mathrm{p}<0.05, *=$ significant, ns=non-significant 
TABLE (2): Results of clinical periodontal outcomes in both studied groups throughout the experimental period.

\begin{tabular}{lcccc}
\hline & Baseline & 3 months & 6 months & P-value \\
\hline GT mean $( \pm$ SD) & & & & \\
SCTG & $1.12( \pm 0.22)$ & $2.57( \pm 0.67)$ & $3.07( \pm 0.65)$ & $0.003^{*}$ \\
PRF & $1.10( \pm 0.36)$ & $2.50( \pm 0.63)$ & $2.13( \pm 0.46)$ & $0.004^{*}$ \\
Mean difference CI (95\%) & $0.02[-0.38,0.41]$ & $0.07[-0.77,0.9]$ & $0.94[0.2,1.67]$ & \\
P-value & $0.925 \mathrm{~ns}$ & $0.862 \mathrm{~ns}$ & $0.016^{*}$ & \\
\hline KTW mean $( \pm$ SD) & & & \\
SCTG & $4.17( \pm 1.17)$ & $4.23( \pm 1.13)$ & $4.27( \pm 1.16)$ & $0.097 \mathrm{~ns}$ \\
PRF & $3.97( \pm 0.85)$ & $3.67( \pm 1.21)$ & $3.67( \pm 1.21)$ & $0.717 \mathrm{~ns}$ \\
Mean difference CI (95\%) & $0.20[-1.13,1.53]$ & $0.56[-0.94,2.07]$ & $0.60[-0.92,2.12]$ & \\
P-value & $0.421 \mathrm{~ns}$ & $0.401 \mathrm{~ns}$ & $0.154 \mathrm{~ns}$ & \\
\hline PES mean $( \pm$ SD) & & & $12.50( \pm 1.05)$ & \\
SCTG & & & $11.33( \pm 0.52)$ & \\
PRF & & & $1.17[0.10,2.23]$ & \\
Mean difference CI (95\%) & & & $0.035^{*}$ & \\
P-value & & & \\
\hline
\end{tabular}

Significance level $p<0.05$, *=significant, $n$ s=non-significant

\section{Patient reported outcomes}

The postoperative pain was reported that the intensity of pain was greater in the SCTG group at different observation times but the difference between groups was not statistically significant $(P$ $\geq 0.05$ ) at days 0 to 7 respectively.

Postoperative swelling as reported by VRS, greater value was noted at the PRF group at the day of the surgery with statistically significant difference $(P=0.034)$. However, in the subsequent observation dates, greater swelling intensities were observed in the SCTG group, with no statistically significant difference between groups $(P \geq 0.05)$ at days 1 to 7 respectively.

Patient satisfaction was measured according to a questionnaire answered by the patients at the end of the follow up period, where the mean difference of patient opinions between both groups showed no statistically significant difference in response to the questionnaire $(P=0.72,0.57$ and 0.53$)$ for questions 1 to 3 respectively.

\section{DISCUSSION}

SCTG has been considered as the gold standard for soft tissue augmentation, yet it still have the disadvantages of an additional harvesting site associated with pain, morbidity and limited amount of graft material ${ }^{[7]}$. Therefore, different alternatives have been suggested such as PRF ${ }^{[31]}$.

PRF gained its popularity as a healing biomaterial for both soft and hard tissue because of the presence of various growth factors. Therefore, PRF could offer several advantages as promoting wound healing, bone growth and maturation, graft 
stabilization and hemostasis and even improving the handling properties of graft material ${ }^{[13]}$.

Standardized intra-oral periapical radiographs with paralleling technique and XCP positioner for assessment of CBL over different time intervals is an accurate method in minimizing distortion and magnification ${ }^{[15,32]}$. CBL was identified immediately after implant placement then at 3,6 and 9 months intervals, since it was proved that $50 \%$ of total crestal bone loss recorded in 12 months period after implant placement ${ }^{[32,33]}$.

The present RCT demonstrated a statistically significant increase in the mean crestal bone loss in both groups at 3,6 and 9 months with no statistically significant difference between the two groups. In the SCTG group the mean crestal bone loss was $1.20( \pm 0.22), 1.37( \pm 0.28)$ and $1.70( \pm 0.20)$ $\mathrm{mm}$ respectively. While in the PRF group the mean crestal bone loss was $0.95( \pm 0.39), 1.20( \pm 0.36)$ and $1.55( \pm 0.15) \mathrm{mm}$ respectively. These results were inferior to an RCT conducted by Wiesner ${ }^{[21]}$ who also augmented soft tissue around delayed implants by SCTG and showed a mean marginal bone loss of $1.14( \pm 0.29) \mathrm{mm}$ after 1 year. Also, less crestal bone loss was expressed in another clinical trial by Zuiderveld et al ${ }^{[34]}$, who achieved $0.42( \pm 0.06) \mathrm{mm}$ and $0.46( \pm 0.04) \mathrm{mm}$ after one month and one-year intervals respectively. The results of the current study agreed with Kenawy et al ${ }^{[35]}$ who obtained mean marginal bone loss $1.0( \pm 0.5), 1.0( \pm 0.5)$ and $1.5( \pm 0.5) \mathrm{mm}$ after 3,6 and 9 months respectively after augmentation with PRF around immediate implants.

Regarding the change in gingival thickness in this trial the mean increase in SCTG group was $1.117( \pm 0.223), 2.567( \pm 0.665)$ and $3.067( \pm 0.650)$ $\mathrm{mm}$ at baseline, 3 and 6 months respectively. While the mean increase in the PRF group was $1.1( \pm 0.358), 2.5( \pm 0.632)$ and $2.133( \pm 0.455) \mathrm{mm}$ respectively. SCTG had statistically significant superior results than PRF after 6 months. This agrees with a study conducted by Wiesner et al ${ }^{[36]}$ who reached a $1.3( \pm 0.61) \mathrm{mm}$ increase in gingival thickness when augmenting using SCTG after one year. Also, this study results were in a line with a randomized controlled clinical trial done by Cairo et al ${ }^{[18]}$, where the buccal gingival thickness showed an increase by $1.2( \pm 0.3) \mathrm{mm}$ in the when using SCTG after 6 months. Marrelli and Tatullo ${ }^{[28]}$, also Hehn et al ${ }^{[37]}$ obtained corresponding results to the current trial, where complete coverage of implants and soft tissue thickness gain between 1-3 $\mathrm{mm}$ were observed after using PRF for soft tissue augmentation around immediate implants.

Regarding the keratinized tissue width, there was a gradual non statistically significant increase from $4.17( \pm 1.17)$, to $4.23( \pm 1.13)$, then $4.27( \pm 1.16)$ $\mathrm{mm}$. While there was a gradual non-statistically significant decrease in the PRF group from $3.97( \pm 0.85) \mathrm{mm}$ at baseline, to $3.67( \pm 1.21) \mathrm{mm}$ at 3 months and remained constant $3.67( \pm 1.21)$ mm 6 months no statistically significant difference between both studied groups. Superior results by Lee et al ${ }^{[38]}$ were found with an increase of more than $3 \mathrm{~mm}$ at 2 years postoperative.

Interestingly, the non-statistically significant decrease in the KTW after 6 months in the PRF group conflicted with a study by Mufti et al ${ }^{[39]}$ who observed an increase in the KTW after using PRF for treating gingival recession. On the other hand, this was previously stated by Jankovic et al ${ }^{[40]}$ who declared that although PRF could enhance wound healing properties yet it did not cause any significant improvement in the KTW when compared to SCTG.

In this trial greater statistically significant values of PES were recorded in the SCTG group when compared to the PRF group after 6 months. This agrees with Wiesner et $\mathrm{al}^{[36]}$ and confirms the fact that SCTG is still the most promising aesthetic graft material around dental implants. This was further proved by Rojo et al. ${ }^{[41]}$.

Concerning the post-operative morbidity and patient satisfaction, although there was no statistically significant difference between both 
treatment modalities, yet it could not be ignored that a secondary surgical site for harvesting the SCTG was annoying for the patient and increased the postoperative pain.

\section{CONCLUSION}

This study demonstrated that although PRF showed less values of crestal bone loss yet both treatment modalities could not prevent the postsurgical crestal bone loss to occur, with no significant difference between them. This study also confirmed the precise role of SCTG in enhancing the gingival thickness and the inability of PRF to compete regarding this parameter. Therefore, further studies with larger samples and longer follow up might be required to clarify other protocols in soft tissue augmentation that might preserve the bone and reduce the crestal loss, enhance the soft tissue and avoid post-operative morbidity after insertion of delayed implants.

\section{REFERENCES:}

1. Henry, P.J., Tooth loss and implant replacement. Aust Dent J, 2000. 45(3): p. 150-72.

2. Ochoa Durand, D. and T. Suzuki, Ochoa D, Suzuki T. Update On Timing Of Implant Placement After Tooth Extraction. Oral Health, Dec 2015. 2015.

3. Buser, D., W. Martin, and U.C. Belser, Optimizing esthetics for implant restorations in the anterior maxilla: Anatomic and surgical considerations. Vol. 19. 2004. 43-61.

4. Kelekis-Cholakis, A., The Importance of Keratinized Tissue Around Implants. Vol. 31. 2015. 102.

5. Al-Sabbagh, M., Implants in the esthetic zone. Dent Clin North Am, 2006. 50(3): p. 391-407, vi.

6. Kao, R.T., M.C. Fagan, and G.J. Conte, Thick vs. thin gingival biotypes: a key determinant in treatment planning for dental implants. J Calif Dent Assoc, 2008. 36(3): p. 193-8.

7. Karthikeyan, B.V., et al., The versatile subepithelial connective tissue graft: a literature update. Gen Dent, 2016. 64(6): p. e28-e33.
8. Lai, P.-C., D. Katwal, and H. Greenwell, Allografts and Xenografts for Periodontal Plastic Surgical Procedures. Current Oral Health Reports, 2019. 6(3): p. 218-229.

9. Dohan, D.M., et al., Platelet-rich fibrin (PRF): a secondgeneration platelet concentrate. Part II: platelet-related biologic features. Oral Surg Oral Med Oral Pathol Oral Radiol Endod, 2006. 101(3): p. e45-50.

10. Pirpir, C., et al., Evaluation of effectiveness of concentrated growth factor on osseointegration. International journal of implant dentistry, 2017. 3(1): p. 7-7.

11. Kawase, T., Platelet-rich plasma and its derivatives as promising bioactive materials for regenerative medicine: basic principles and concepts underlying recent advances. Odontology, 2015. 103(2): p. 126-35.

12. Ezzatt, O.M.,Autologous Platelet Concentrate Preparations in Dentistry. Biomedical Journal of Scientific \& Technical Research, 2018.8(5).

13. Miron, R.J., et al., Platelet-Rich Fibrin and Soft Tissue Wound Healing: A Systematic Review. Tissue Eng Part B Rev, 2017. 23(1): p. 83-99.

14. Wang, C. and X. Ma, Preliminary study on the healing effect of PRF for soft tissue defects in oral implants. Journal of King Saud University - Science, 2020.

15. Gulsahi, A., Bone Quality Assessment for Dental Implants. 2011.

16. Abdelkarim, M., et al., Assessment of bone healing around immediately loading dental implants in posterior maxilla with two different osteotomy techniques. Indian Journal of Multidisciplinary Dentistry, 2015. 5(1): p. 31-39.

17. Boora, P., Effect of Platelet Rich Fibrin (PRF) on Periimplant Soft Tissue and Crestal Bone in One-Stage Implant Placement: A Randomized Controlled Trial. Journal of Clinical and Diagnostic Research, 2015.

18. Cairo, F., et al., Xenogeneic collagen matrix versus connective tissue graft for buccal soft tissue augmentation at implant site. A randomized, controlled clinical trial. J Clin Periodontol, 2017. 44(7): p. 769-776.

19. Furhauser, R., et al., Evaluation of soft tissue around single-tooth implant crowns: the pink esthetic score. Clin Oral Implants Res, 2005. 16(6): p. 639-44.

20. Garcia, B., et al., Pain and swelling in periapical surgery. A literature update. Med Oral Patol Oral Cir Bucal, 2008. 13(11): p. E726-9. 
21. Wiesner, G., et al., Connective tissue grafts for thickening peri-implant tissues at implant placement. One-year results from an explanatory split-mouth randomised controlled clinical trial. Vol. 3. 2010. 27-35.

22. Augustin, G., et al., Thermal osteonecrosis and bone drilling parameters revisited. Arch Orthop Trauma Surg, 2008. 128(1): p. 71-7.

23. Nagarajan, B., et al., Evaluation of Crestal Bone Loss Around Implants Placed at Equicrestal and Subcrestal Levels Before Loading: A Prospective Clinical Study. J Clin Diagn Res, 2015. 9(12): p. ZC47-50.

24. Turkyilmaz, I. and E.A. McGlumphy, Influence of bone density on implant stability parameters and implant success: a retrospective clinical study. BMC Oral Health, 2008. 8: p. 32.

25. Langer, B. and L. Calagna, The subepithelial connective tissue graft. J Prosthet Dent, 1980. 44(4): p. 363-7.

26. Hurzeler, M.B. and D. Weng, A single-incision technique to harvest subepithelial connective tissue grafts from the palate. Int J Periodontics Restorative Dent, 1999. 19(3): p. 279-87.

27. Zeltner, M., et al., Randomized controlled clinical study comparing a volume-stable collagen matrix to autogenous connective tissue grafts for soft tissue augmentation at implant sites: linear volumetric soft tissue changes up to 3 months. J Clin Periodontol, 2017. 44(4): p. 446-453.

28. Marrelli, M. and M. Tatullo, Influence of PRF in the healing of bone and gingival tissues. Clinical and histological evaluations. Eur Rev Med Pharmacol Sci, 2013. 17(14): p. 1958-62.

29. Arora, S., et al., A Comparative Evaluation of Immediate Implant Placement in Fresh Extraction Socket with and without the Use of Platelet-rich Fibrin: A Clinical and Radiographic Study. International Journal of Oral Implantology \& Clinical Research, 2016. 7: p. 48-58.

30. Kenawy, M.H.E., U.M. El Shinnawi, and A.M.S.a.F.H. Ahmed, Efficacy of platelet rich fibrin (PRF) membrane in immediate dental implant. Mans J Dent, 2014. 1: p. 78-84.

31. Fu, J.H., C.Y. Su, and H.L. Wang, Esthetic soft tissue management for teeth and implants. J Evid Based Dent Pract, 2012. 12(3 Suppl): p. 129-42.
32. Hermann, J.S., et al., Crestal bone changes around titanium implants: a methodologic study comparing linear radiographic with histometric measurements. Int $\mathrm{J}$ Oral Maxillofac Implants, 2001. 16(4): p. 475-85.

33. Nandal, S., P. Ghalaut, and H. Shekhawat, A radiological evaluation of marginal bone around dental implants: An invivo study. National journal of maxillofacial surgery, 2014. 5(2): p. 126-137.

34. Zuiderveld, E.G., et al., Effect of connective tissue grafting on peri-implant tissue in single immediate implant sites: A RCT. J Clin Periodontol, 2018. 45(2): p. 253-264.

35. Kenawy, M.H.E., U.M. El Shinnawi, and A.M.S.a.F.H. Ahmed, Efficacy of platelet rich fibrin (PRF) membrane in immediate dental implant. Mansoura Journal of Dentistry, 2014. 1(3): p. 78-84.

36. Wiesner, G., et al., Connective tissue grafts for thickening peri-implant tissues at implant placement. One-year results from an explanatory split-mouth randomised controlled clinical trial. Eur J Oral Implantol, 2010. 3(1): p. 27-35.

37. Hehn, J., et al., The effect of PRF (platelet-rich fibrin) inserted with a split-flap technique on soft tissue thickening and initial marginal bone loss around implants: results of a randomized, controlled clinical trial. Int J Implant Dent, 2016. 2(1): p. 13.

38. Lee, J.W., et al., Restoration of a peri-implant defect by platelet-rich fibrin. Oral Surg Oral Med Oral Pathol Oral Radiol, 2012. 113(4): p. 459-63.

39. Mufti, S., et al., Comparative Evaluation of Platelet-Rich Fibrin with Connective Tissue Grafts in the Treatment of Miller's Class I Gingival Recessions. Contemp Clin Dent, 2017. 8(4): p. 531-537.

40. Jankovic, S., et al., Use of platelet-rich fibrin membrane following treatment of gingival recession: a randomized clinical trial. Int J Periodontics Restorative Dent, 2012. 32(2): p. e41-50.

41. Rojo, E., et al., Soft tissue volume gain around dental implants using autogenous subepithelial connective tissue grafts harvested from the lateral palate or tuberosity area. A randomized controlled clinical study. Journal of Clinical Periodontology, 2018. 45. 